Proceedings of the

Combustion Institute

\title{
A numerical study on the instability of oblique detonation waves with a two-step induction-reaction kinetic model
}

\author{
Pengfei Yang ${ }^{a, b, c}$, Honghui Teng ${ }^{\mathrm{a}, \mathrm{b}, *}$, Hoi Dick Ng ${ }^{\mathrm{d}}$, Zonglin Jiang b,c \\ ${ }^{a}$ School of Aerospace Engineering, Beijing Institute of Technology, Beijing 100081, China \\ ${ }^{\mathrm{b}}$ State Key Laboratory of High Temperature Gas Dynamics, Institute of Mechanics, Chinese Academy of Sciences, Beijing \\ 100190, China \\ ${ }^{\mathrm{c}}$ School of Engineering Sciences, University of Chinese Academy of Sciences, Beijing 100049, China \\ ${ }^{\mathrm{d}}$ Department of Mechanical, Industrial and Aerospace Engineering, Concordia University, Montreal, QC H3G 1 M8, \\ Canada
}

Received 24 November 2017; accepted 17 May 2018

Available online xxx

\begin{abstract}
In this study, the surface instability of oblique detonation waves (ODW) formed by two-dimensional, semi-infinite wedges is investigated numerically by solving the unsteady Euler equations with a two-step induction-reaction kinetic model. The chemical kinetic model introduces two length scales, namely, induction and reaction lengths, which can be varied independently to change the sensitivity of the chemical reaction and also the shape of the reaction zone structure. The present numerical results elucidate that both smooth and cellular ODW surfaces may appear after the initiation, and the surface becomes unstable when the reaction zone length decreases while keeping the induction zone the same as observed in normal detonation wave propagation. To investigate the degree of instability quantitatively, the oscillations of post-shock pressure inside the reaction zone are examined, and analyzed using Fast Fourier Transformation (FFT) to get the power spectral density (PSD). Results suggest that there are two types of unstable surfaces, one is dominated by random disturbances, without distinct large amplitude unstable modes, on the ODW surface due to the upstream perturbations interacting with the incoming flow and continuous generation within the structure, while the other formed from the inherent disturbances convected from upstream in the initiation region and later developed into dominant unstable modes via an apparent bifurcation pattern. Equivalent to normal detonations, the stability parameter $\chi$ as defined by the ratio of induction length over the reaction length
\end{abstract}

* Corresponding author at: School of Aerospace Engineering, Beijing Institute of Technology, Beijing 100081, China. E-mail address: hhteng@bit.edu.cn (H. Teng). 
multiplied by the global reduced activation energy can also be used to describe qualitatively the trends of the ODW surface instability observed in this study.

(C) 2018 The Combustion Institute. Published by Elsevier Inc. All rights reserved.

Keywords: Oblique detonation; Induction-reaction kinetics; Unstable surface; Power spectral density

\section{Introduction}

The concept of using oblique detonation waves (ODW) for high efficiency propulsion systems has generated great interest by their potential use in the development of air-breathing hypersonic aircraft [1-6]. This class of propulsion engines not only achieves a high thermal cycle efficiency by detonations, but also shares advantages with ramjet engines. To advance development of ODW-based engines, it is vital to understand the fundamental ODW structure and its dynamics. The pioneering work by $\mathrm{Li}$ et al. [7] revealed that the multidimensional oblique detonation structure consists of a non-reactive oblique shock, an induction region, a set of deflagration waves, and an oblique detonation surface all united at a multi-wave point. Such structure was later confirmed by experiments [8]. Parametric studies were performed numerically to investigate the wave structures on various parameters, such as the inflow Mach number, wedge angle and the reactivity of the mixture [9-11]. Besides the two-dimensional, wedged-induced ODW, experimental and numerical studies were performed on the cone-induced ODW to investigate the effects of Taylor-Maccoll flow $[12,13]$. Furthermore, ODW induced by blunt projectiles has been studied experimentally, illustrating the complicated structures concerning the interaction of curved shock/detonation and expansion wave [14-17]. Effects of the inflow equivalence ratio inhomogeneity on ODW structures, featured by the distorted reaction surfaces, were also studied aiming to further the engine application [18-20].

Another fundamental characteristic of ODW is the instability of the formed detonation. Cellular ODW surfaces with triple points have been demonstrated by numerical simulations [10] and observed experimentally as well [21]. It is found that combustible mixtures with large value of activation energy are more susceptible to transverse waves or triple point formation [10]. Choi et al. [22] showed several cellular structures with different activation energies and pointed out that these structures cannot be captured without enough numerical grid resolution. By using high-order numerical schemes and extending the computational domain sufficient for the instability to develop, recent numerical simulations indeed reveals the existence of cellular structures and their evolution processes $[23,24]$.
The formation mechanisms of these cellular structures are fundamental features important to further exploring the propagation dynamics of ODWs. Our previous study [25] found that the triple points derived from the amplification of the upstream disturbance, and high degree of overdrive will delay but not eliminate the amplification. However, there is a concern whether the simulated phenomenon is physical or a numerical artifact of the single-step chemistry model due to its simple kinetic description. To provide a compromise on the detailed reaction mechanism, a two-step induction-reaction kinetic model, consisting of a thermally neutral induction step followed by a main heat release reaction layer, can be considered. Such a model allows the introduction of two length scales, i.e., induction and reaction lengths, which can be varied independently to change the sensitivity of the chemical reaction and also the shape of the reaction zone structure. Recent studies [2629] have revealed that the dynamics of the detonation structure is strongly governed not only by the global reaction temperature sensitivity, but also the lengths of induction and main heat release layer.

Motivated by the aforementioned studies, numerical simulations are performed using a twostep chemical kinetic model to analyze the instability of oblique detonation waves induced by a two-dimensional (2-D), semi-infinite wedge. The effects of heat release rate are discussed and the stability parameter $\chi$, proposed previously in the investigation of normal detonations [27], is explored for quantifying the results. Quantitative analysis is also performed by examining the oscillations of post-shock pressure evolution, and determining the corresponding power spectral density (PSD) using Fast Fourier Transformation (FFT) to reveal the instability features. Different types of unstable surfaces are demonstrated and discussed.

\section{Computational details}

A schematic of a typical ODW induced by a 2-D, semi-infinite wedge is given in Fig. 1. The presence of the wedge in a supersonic combustible inflow induces first an oblique shock wave (OSW). For a high inflow Mach number causing a high post-shock temperature behind the OSW, an exothermic chemical reaction begins, leading to the ODW formation. As in our previous 


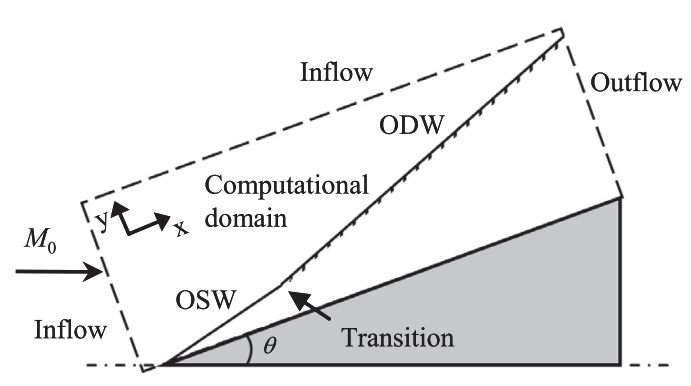

Fig. 1. Schematic of a typical ODW.

computations $[13,20,24,25]$, the coordinate is rotated to the direction along the wedge surface and the Cartesian grid in the rectangular domain enclosed by the dashed line in Fig. 1 is aligned with the wedge surface.

Following previous studies, e.g., [10, 11,22-25], the reactive Euler equations are used as governing equations for modeling the ODW flow field. To implement the two-step model for modeling chain-branching kinetics [27], two additional reaction variables are introduced: one for the induction, $\xi$, and the other for the heat release, $\lambda$. The transport equations of these reaction variables are:

$$
\begin{aligned}
\frac{\partial(\rho \xi)}{\partial t} & +\frac{\partial(\rho u \xi)}{\partial x}+\frac{\partial(\rho v \xi)}{\partial y} \\
& =H(1-\xi) \rho k_{I} \exp \left[E_{I}\left(\frac{1}{T_{S}}-\frac{1}{T}\right)\right]
\end{aligned}
$$

$$
\begin{aligned}
\frac{\partial(\rho \lambda)}{\partial t} & +\frac{\partial(\rho u \lambda)}{\partial x}+\frac{\partial(\rho \nu \lambda)}{\partial y} \\
& =[1-H(1-\xi)] \rho(1-\lambda) k_{R} \exp \left[-\frac{E_{R}}{T}\right]
\end{aligned}
$$

with the Heaviside step function given by:

$H(1-\xi)= \begin{cases}1, & \text { if } \xi \leq 1 \\ 0, & \text { if } \xi>1\end{cases}$

The specific total energy is thus expressed as:

$$
e=\frac{p}{\rho(\gamma-1)}+\frac{1}{2}\left(u^{2}+v^{2}\right)-\lambda Q
$$

The variables $\rho, u, v, p, e$ and $Q$ are the density, $x$ - direction velocity, $y$-direction velocity, pressure, specific total energy, and the amount of chemical heat release, respectively. All the variables have been non-dimensionalized by reference to the uniform unburned state as follows:

$$
\rho=\frac{\tilde{\rho}}{\rho_{0}}, p=\frac{\tilde{p}}{p_{0}}, T=\frac{\tilde{T}}{T_{0}}, u=\frac{\tilde{u}}{\sqrt{R T_{0}}}
$$

The dispersion controlled dissipation scheme [30] together with a 3rd order Runge-Kutta algorithm are used to approximate the solution of the
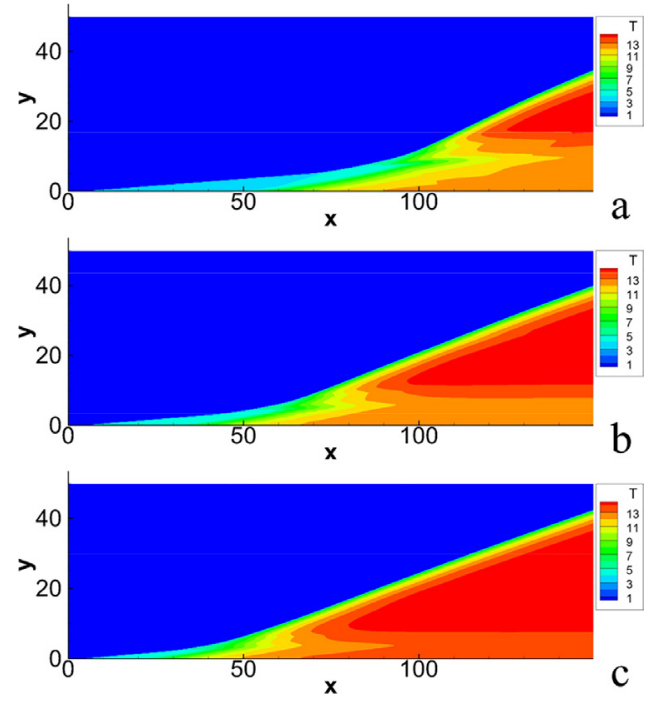

Fig. 2. Temperature field of oblique detonations with $k_{R}=1.0$ and $\theta=28^{\circ}(\mathrm{a}), 30^{\circ}(\mathrm{b})$, and $32^{\circ}$ (c).

governing equations. The main parameters are set to be

$Q=50, \gamma=1.2, E_{I}=5.0 T_{S}, E_{R}=1.0 T_{S}$

where $T_{s}$ is the post-shock temperature of Chapman-Jouguet (CJ) detonation. This parameter set represents the combustible gas with relatively large heat release and modest activation energy [27]. There are two key pre-exponential factors, $k_{\mathrm{I}}$ and $k_{\mathrm{R}}$, which are used to complete the chemistry model. In this study, $k_{\mathrm{I}}=-u_{v n}$ where $u_{v n}$ is the post-shock particle velocity in the shockfixed frame for the corresponding $\mathrm{CJ}$ detonation, whereby the induction length of the $\mathrm{CJ}$ detonation is fixed to unity. The factor of the heat release step $k_{R}$ is employed as the bifurcation parameter to vary the reaction length scale of the main heat release layer. The inflow Mach number $M_{o}$ is fixed to be 10 and the wedge angle $\theta$ is varied to get different structural configurations of ODW.

\section{Numerical results and discussion}

\subsection{Basic ODW structure and resolution study}

The test cases with $k_{R}=1.0$ and $\theta=28^{\circ}, 30^{\circ}$, and $32^{\circ}$ are first simulated to illustrate the basic structure of ODW, as shown in Fig. 2. Initially the whole flow field has uniform density, velocity and pressure; both the density and pressure are unity as the unburned state, and the velocity is calculated and projected according to $M_{o}$ and $\theta$. The left and upper boundary conditions are the inflow one, where the parameters are fixed to be constant for the supersonic flow. Outflow conditions are 

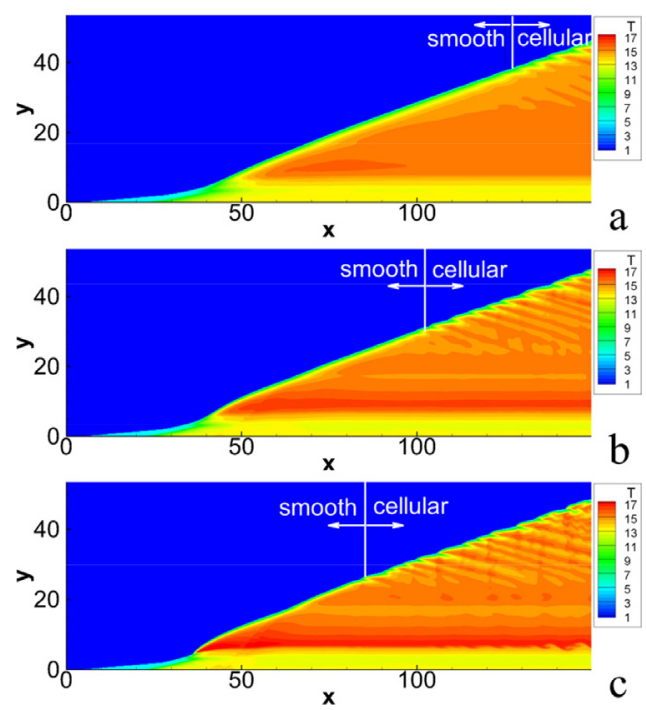

Fig. 3. Temperature field of oblique detonations with $\theta=32^{\circ}$ and $k_{R}=2.0(\mathrm{a}), 3.0(\mathrm{~b})$, and 4.0 (c).

extrapolated from the interior, implemented on the right and lower boundaries before the wedge. Slip boundary conditions are used on the wedge surface, which starts from $x=5$ on the lower boundary. The default grid scale is 0.050 which will be verified in the later resolution study. From Fig. 2, it is observed that the wedge angle will change the initiation position, e.g., increasing $\theta$ makes the ODW structure to move upstream, resulting from the raised post-oblique shock temperature. The ODW surface is smooth, without cellular structures observed, in these cases within the present computational domain. This kind of structures has been observed in previous studies (e.g., [20,24]) and verified in [31], although different chemistry models are used. Multidimensional gaseous detonations are inherently unstable, however unstable modes can be suppressed by the ODW overdrive or small reaction sensitivity. As such, smooth ODW surfaces are observed even within a large computational domain $[20,22,23]$. Depending on the chemical kinetics effect, weak wrinkles may manifest but take an extremely long evolution to appear [25]. Nevertheless, smooth surface segments often remain within a practical computational domain worthwhile for a fundamental study on the basic ODW structure without the instability effect [31].

The cellular structures are observed in Fig. 3, by increasing $k_{R}$ while keeping $\theta=32^{\circ}$ in all cases. For $k_{R}=2.0$ in Fig. 3a, the triple points are only generated near the outflow boundary. Further increase of $k_{R}$ to 3.0 enhances the instability, inducing more triple points in the computational domain. When $k_{R}=4.0$ in Fig. 3c, the instability manifests at a faster growth rate and the cellular surface takes
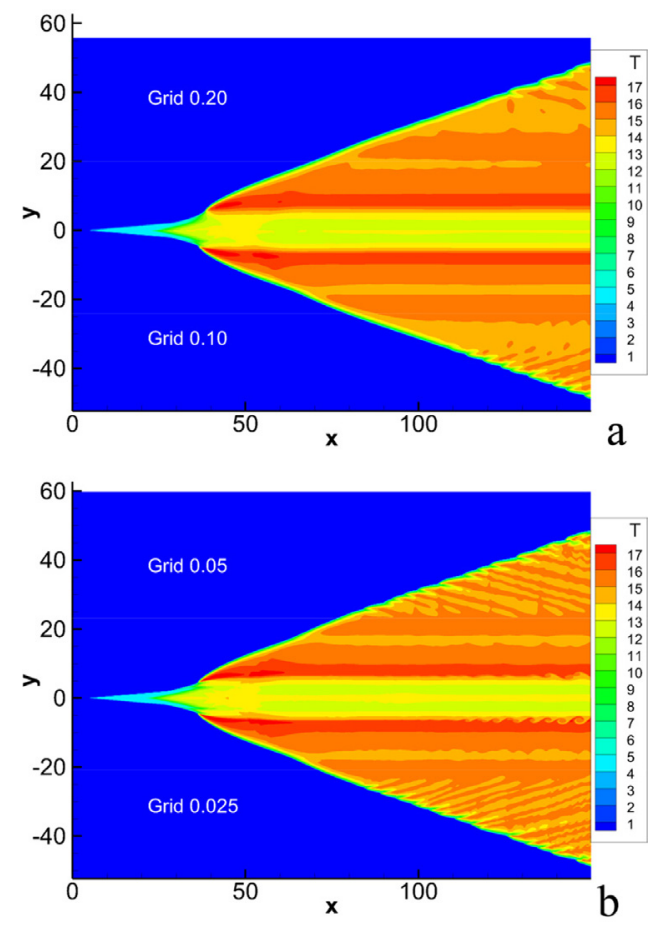

Fig. 4. Temperature field of oblique detonations with $\theta=32^{\circ}$ and $k_{R}=4.0$, (a) grid 0.020 (upper) and 0.010 (lower); (b) grid 0.05 (upper) and 0.025 (lower).

over half length of the surface with only a small section of smooth surface after the initiation. To ensure the ODWs reach their final steady positions, the ODW angles are measured from the numerical results. These agree with the theoretical ODW angle of $51.14^{\circ}$ determined using polar analysis within $\pm 0.1^{\circ}$. Furthermore, more complex flow field, developed from the initial cellular structure, is observed near the outflow boundary as shown in Fig. 3c. These phenomena are in good agreement with the finding from previous works using the single-step chemistry model $[22,24]$.

To verify the effect of computational grids, a resolution study is performed. The ODW structure shown in Fig. 3c, i.e., the most unstable case considered in this study with $\theta=32^{\circ}$ and $k_{R}=4.0$, is used for comparison. Figure 4 shows the results using different grid sizes. The default grid 0.05 corresponds to about 20 points per the induction length of the $\mathrm{CJ}$ detonation. By decreasing the grid scale of $0.20-0.05$, the location for the onset of instability moves upstream, but keeps the same when the grid scale decreases further to 0.025 . In Fig. $4 b$, both flow fields are almost the same with each other, especially near the initiation region. It is worth noting that after the formation of cellular structure, it becomes difficult to get the exact same flow fields on the detonation surface due to 


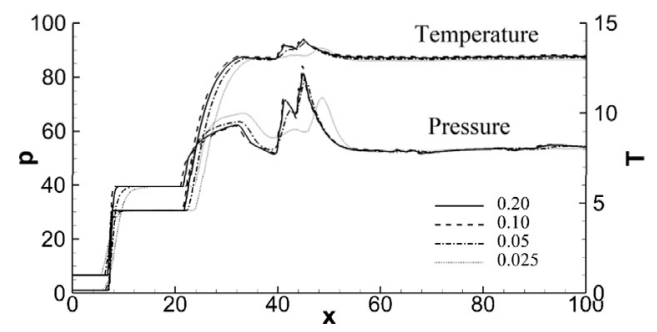

Fig. 5. Pressure and temperature on the wedge with different grid scales.

a certain degree of randomness of the instability. Nevertheless, both results show good agreement in term of the location for the onset of instability and the overall unstable features. For further comparison, pressure and temperature on the wedge, i.e., along the line $y=0$, are plotted in Fig. 5. From upstream to downstream, the pressure/temperature rises induced by the oblique shock wave, the reaction front, and the shock in the product can be observed successively. The pressure/temperature curves from different grids are almost overlapped together except for the coarsest one of 0.20 . Hence, the default size 0.05 is deemed sufficient to capture the main physical and chemical processes, and used in all later simulations.

\subsection{The effect of heat release rate}

The results shown in Fig. 3 indicate qualitatively that increasing $k_{R}$ will render the ODW surface more unstable. Furthermore, increasing $k_{R}$ not only induces the cellular surface, but also influences the oblique shock-to-detonation transition due to its consequence of faster heat release rate. The transition position moves upstream, and the initiation structure changes from the smooth one (by a curved shock) into the abrupt one (by a multi-wave point). Therefore, the heat release rate is an important factor to determine the wave structure of the initiation region and its location.

To analyze quantitatively the ODW instability, post-shock pressure oscillations at the half reaction zone are recorded when the ODW flow fields are quasi-steady, i.e., the ODW surface reaches its final position. It is worth noting that due to a different flow nature, the degree of ODW instability is assessed by not only the spectrum of unstable modes at different frequencies (i.e., degree of chaotic fluctuations or cell irregularity as in normal detonations), but also the magnitude of the oscillation as well as the power spectrum level. A strongly unstable ODW is thus defined as an unstable surface characterized by instabilities amplified into multiple dominant unstable modes, where a weakly unstable ODW can also be characterized by irregular fluctuations but with relatively weak amplitude.

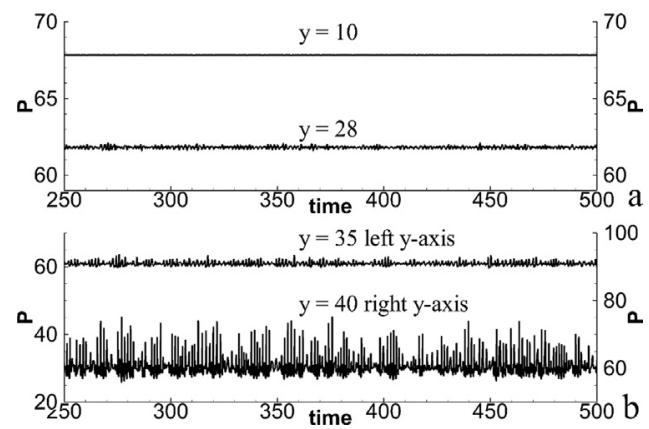

Fig. 6. Post-shock pressure oscillation at the half reaction zone with $k_{R}=2.0$ and $\theta=32^{\circ}$.

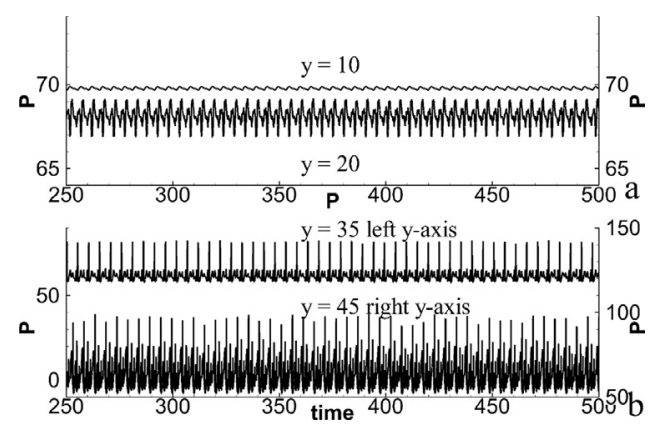

Fig. 7. Post-shock pressure oscillation at the half reaction zone with $k_{R}=4.0$ and $\theta=32^{\circ}$.

Two cases are studied, one is the case with $k_{R}=2.0$ and $\theta=32^{\circ}$, and the other one with $k_{R}=4.0$ and $\theta=32^{\circ}$. From Fig. 3 , the former has a long initial smooth surface, representing the weakly unstable ODW, and the latter generates promptly the cellular structure, representing the strongly unstable ODW. The oscillations of the weakly unstable ODW at several key positions are shown in Fig. 6, whose flow fields are demonstrated in Fig. 3a. On the line $y=10$, the initiation just occurs and the pressure oscillation cannot be observed. The weak oscillation can be observed on the line $y=28$ and becomes strong on the line $y=35$. From $y=35$ to 45 , the oscillation become much more apparent, but there are no clear regular, dominant patterns observed for the settlement of the final ODW surface, illustrating a type of random, weak oscillations without obvious orders.

Figure 7 shows the oscillations of the strongly unstable ODW, whose flow fields are demonstrated in Fig. 3c. Overall, Fig. 7 illustrates a different oscillation phenomenon compared to that of Fig. 6 . As soon as the ODW is initiated, oscillations with small amplitude are observed, and also featured by a single-period mode. On the line $y=20$ where the cellular structures have not yet appeared, a twopeak oscillation mode forms with a constant time cycle about 5 , showing very regular patterns. On 


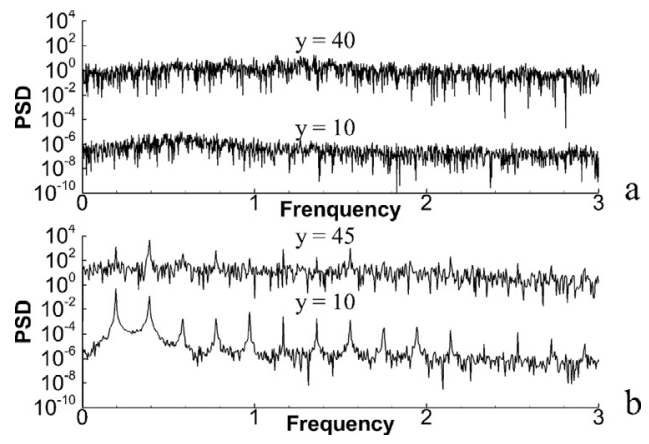

Fig. 8. Power spectral density of different lines with $k_{R}=2.0$ (a) and 4.0 (b) using Fast Fourier Transformation.

the lines $y=35$ and 45 where the cellular structures have appeared, the oscillation is dominated by one high spike with several low ones. Nevertheless, the cycle time between two subsequent dominant peaks keeps the same as the upstream ones, showing a clear oscillation pattern derived from the upstream disturbance. The development of this instability feature follows a series of bifurcations that such route to chaos leads to establish the final unstable ODW structure [32].

The post-shock pressure oscillation is essentially a time series, from which the power spectral density (PSD) can be computed, see Fig. 8. It is obtained via a FFT (Fast Fourier Transformation) of the autocorrelation sequence of the time series, which can be considered as the distribution of the power over frequencies. As shown in Fig. 8, the strongly unstable ODW has several dominant frequencies, with the interval about 0.2 , while the weakly unstable ODW has no dominant frequencies. For the strongly unstable ODW, the dominant frequencies have already appeared on the line $y=10$, which persist and do not change even after the cellular structure appears. As shown in Fig. 8b, the PSD amplifications vary as high as five orders, but essentially all the dominant frequencies keep the same. Therefore, the evolution of strongly unstable ODW can be viewed as the amplification of the upstream inherent disturbances. The unstable modes originated from the initiation region persist and evolve via a bifurcation process to establish the cellular ODW surface. In contrast, in the weakly unstable ODW, the weak instabilities derive from the upstream disturbances or continuous, random generation within the flow field without any regular or predictable spatiotemporal order. These perturbations generate irregular fluctuations of the ODW surface, but are unable to develop into multiple nonlinear instability modes with large magnitude.
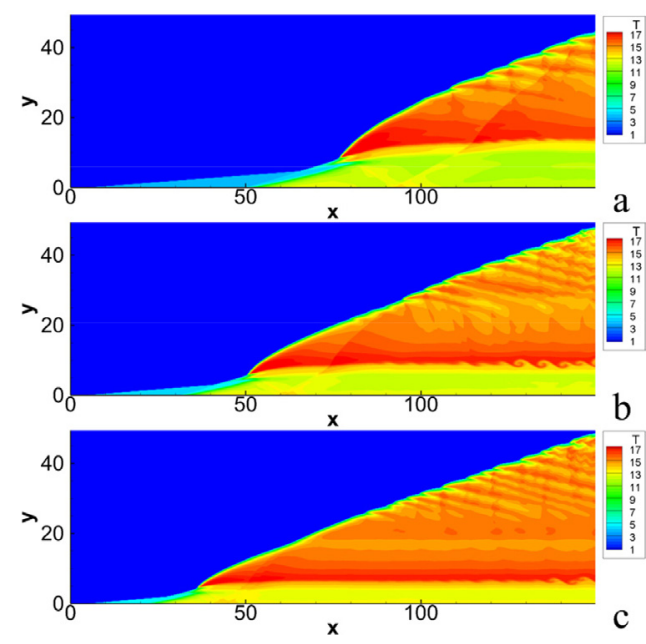

Fig. 9. Temperature field of oblique detonations with $k_{R}=4.0$ and (a) $\theta=28^{\circ}$; (b) $30^{\circ}$; and (c) $32^{\circ}$.
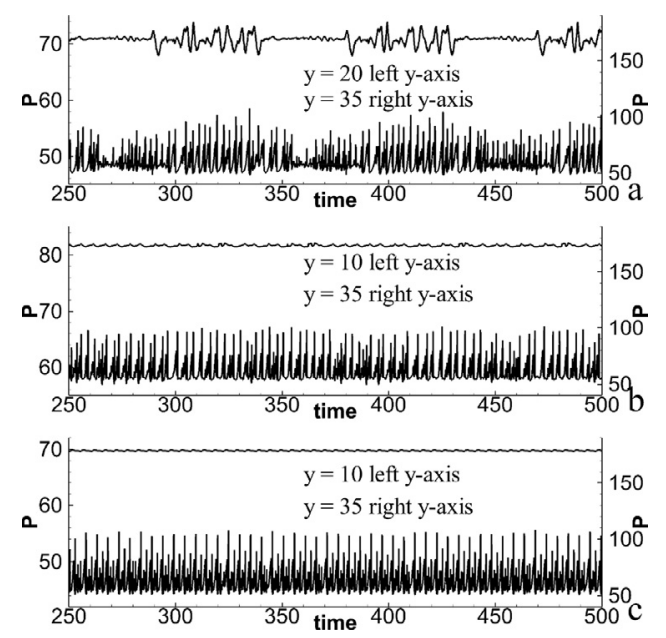

Fig. 10. Post-shock pressure oscillation at the half reaction zone with $k_{R}=4.0$ and (a) $\theta=28^{\circ}$; (b) $30^{\circ}$; and (c) $32^{\circ}$.

\subsection{The influence of wedge angle}

To further investigate the ODW instability with different heat release rates, more cases with different wedge angles are simulated. The flow structures with $k_{R}=4.0$ and $\theta=28^{\circ}, 30^{\circ}$ and $32^{\circ}$, are shown in Fig. 9. The post-shock pressure history and the corresponding PSD for the pressure oscillations at half reaction zones are given in Figs. 10 and 11.

From these results, it is shown that decreasing $\theta$ causes the final ODW waves more unstable, showing a wider range of irregular, large amplitude fluctuations. For the low wedge angle of $\theta=28^{\circ}$, a spectrum of unstable modes (multi-modes) is shown in the PSD. By increasing $\theta$, the oscillations 


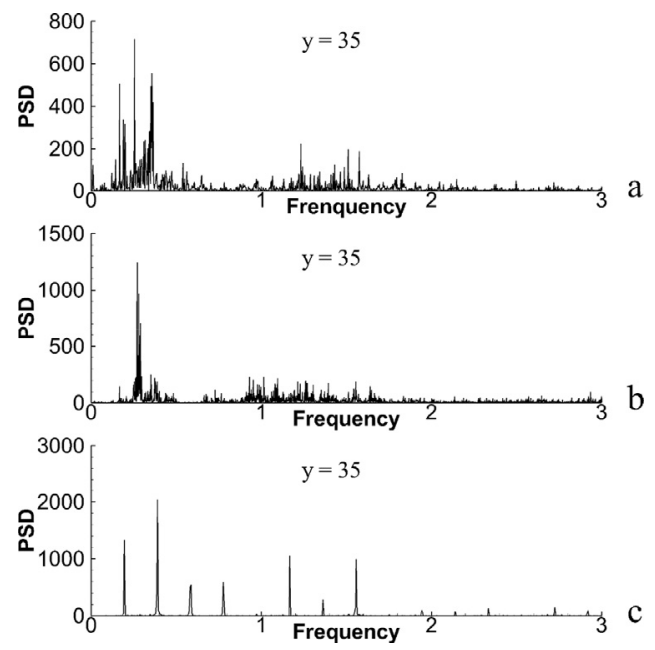

Fig. 11. Power spectral density at cellular surfaces with $k_{R}=4.0$ and (a) $\theta=28^{\circ}$; (b) $30^{\circ}$; and (c) $32^{\circ}$ using Fast Fourier Transformation.

are characterized by fewer modes. At $\theta=32^{\circ}$, the PSD shows primarily a dominant frequency approaching to a more regular oscillation. By decreasing $\theta$ from $32^{\circ}$ to $28^{\circ}$, the ODW overdriven degree also decreases from 1.57 to 1.37 . Consequently, the post-shock temperature behind the ODW is lower, rendering the combustible mixture more sensitive to temperature fluctuation. Such behavior is clearly demonstrated from the present numerical results. Decreasing $\theta$ also reduces the strength of the oblique shock and increases the induction time for the reaction, resulting in a downstream shift of the initiation point or the onset of ODW.

\subsection{The validity of stability parameter $\chi$}

In $[27,29]$, the cellular irregularity of a detonation propagating in a tube has been characterized quantitatively by proposing the stability parameter $\chi$ defined as:

$\chi=\frac{E_{I}}{T_{S}} \frac{\Delta_{I}}{\Delta_{R}}$

where $\Delta_{I}$ and $\Delta_{R}$ denote the characteristic induction and reaction length, respectively. It is found that the degree of instability of normal detonations, both one-dimensional pulsating detonation and 2-D cellular detonation, can be classified by $\chi$. The larger the $\chi$, the more irregular is the detonation structure. Benefiting from the advantages of the two-step kinetic model, i.e., with well-defined induction and reaction length, a quantitative assessment based on $\chi$ can be performed. To ascertain whether $\chi$ is applicable for quantifying instability of oblique detonations, $\chi$ as a function of $k_{R}$ and $\theta$ is shown in Table $1 . \Delta_{I}$ and $\Delta_{R}$ are those perpendicular to wave surfaces, and $\chi$ is calculated
Table 1

Stability parameter $\chi$ in the cases of different $\theta$ and $k_{R}$ values.

\begin{tabular}{llll}
\hline$\chi$ & $\theta=28^{\circ}$ & $\theta=30^{\circ}$ & $\theta=32^{\circ}$ \\
\hline$k_{R}=1.0$ & 0.97 & 0.94 & 0.92 \\
$k_{R}=2.0$ & 1.92 & 1.88 & 1.82 \\
$k_{R}=3.0$ & 2.86 & 2.80 & 2.72 \\
$k_{R}=4.0$ & 3.79 & 3.71 & 3.61 \\
\hline
\end{tabular}

from shock/detonation polar relations, neglecting the curved ODW surface near the initiation region. Recall that in this study the definitions of weakly and strongly unstable ODWs take into account not only the irregularity of the unstable surface but also the bifurcation of multiple dominant nonlinear unstable modes. By increasing $k_{R}$ or decreasing $\theta$, the value of $\chi$ increases. The increase of $\chi$ is found to agree with the present results (see Figs. 3 and 9) that the surface becomes more unstable with multiple dominant mode oscillations.

The stability parameter $\chi$ stems from the coherence energy release concept. For large $\chi$, the resulting incoherence between neighboring power pulses due to high reaction sensitivity provides the necessary chemico-gasdynamic interactions for the development of large amplitude, irregular instability [27]. For ODWs, mixtures with large $\chi$ induce the upstream disturbance convected downstream to develop into distinct instability modes. In contrary, for small $\chi$, where the exothermicity profile remains smooth and coherent to any disturbance, any perturbation will remain weak or the number of unstable modes will decrease leading to a more regular unstable ODW surface.

The stability parameter $\chi$ thus appears to describe qualitatively the instability trends observed for ODW surfaces. Nevertheless, to verify the applicability of $\chi$ for a more quantitative assessment, additional flow parameter sets and combustion characteristics are required for future works.

\section{Conclusion}

Although the cellular instability of ODWs has long been observed numerically and experimentally, there remain outstanding issues concerning its dynamic mechanism and evolution. To investigate systematically the ODW instability, a two-step model is employed in this work to mimic the induction-reaction kinetic processes. Using the numerical results, we perform the nonlinear dynamic analysis to investigate the unstable ODW surface, its degree of instability and bifurcation phenomena.

By increasing the pre-exponential factor of the main heat release rate $k_{R}$, the ODW surface becomes unstable, characterized by multi-mode, large amplitude oscillations. To explore the instability 
features and quantify the present numerical results, the oscillations of post-shock pressure at the halfreaction zone length are recorded and examined. The PSD estimates using FFT are used to analyze the evolution of dominant unstable modes and the origins of disturbances. It is found that for the low $k_{R}$ case, weak oscillations are resulted from the existence of upstream perturbations or continuous random disturbances in the flow field without any clear pattern, while for the prominent unstable case with high $k_{R}$ instability is developed from amplification of upstream inherent disturbances generated in the initiation region toward dominant modes characterizing the final unstable ODW surface. These results demonstrate distinguished instability features of oblique detonations. It is worth noting that recent studies demonstrate that the initiation structures may also involve a transient, oscillating process [33-35]. The coupling between the oscillating initiation structure and the subsequent ODW surface instability needs more attention in future study.

Previous investigations on the unstable ODW find that the formation of triple points is dependent on the wedge angle, effective activation energy, and so on $[10,22-25]$. By taking advantage of the twostep reaction model, this study examines the stability parameter $\chi$ to describe the instability trends of oblique detonations. For ODWs, increasing $k_{R}$ or decreasing $\theta$ leads to an increase in $\chi$. Equivalent to normal detonations, the effect of increasing $\chi$ agrees with the simulation that the surface becomes more unstable with multi-mode, large amplitude oscillations.

\section{Acknowledgment}

The research is supported by the National Natural Science Foundation of China NSFC (Nos. 91641130 and 11372333) and the Natural Sciences and Engineering Research Council of Canada (NSERC).

\section{Reference}

[1] K. Kailasanath, AIAA J. 41 (2003) 145-159.

[2] P. Wolanski, Proc. Combust. Inst. 34 (2013) 125-158.

[3] A.J. Higgins, J. Propul. Power 22 (2006) 1170-1187.

[4] D.T. Pratt, J.W. Humphrey, D.E. Glenn, J. Propul. Power 7 (1991) 837-845.

[5] J.M. Powers, in: J. Buckmaster, T.L. Jackson, A. Kumar (Eds.), Combustion in High Speed Flows, Springer, Netherlands, 1994, pp. 345-371

[6] G.P. Menes, H.G. Adelman, J.L. Cambier, J.V. Bowles, J. Propul. Power 8 (1992) 709-713.

[7] C. Li, K. Kailasanath, E.S. Oran, Phys. Fluids 6 (1994) 1600-1611.
[8] C. Viguier, L.F. Figueira da Silva, D. Desbordes, B. Deshaies, Symp. (Int.) Combust. 26 (2) (1996) 3023-3031

[9] L. da Silva Figueira, B. Deshaies, Combust. Flame 121 (2000) 152-166.

[10] M.V. Papalexandris, Combust. Flame 120 (2000) 526-538.

[11] H.H. Teng, Z.L. Jiang, J. Fluid Mech. 713 (2012) 659-669.

[12] J. Verreault, A.J. Higgins, Proc. Combust. Inst. 33 (2) (2011) 2311-2318.

[13] P. Yang, H.D. Ng, H. Teng, Z. Jiang, Phys. Fluids 29 (8) (2017) 086104.

[14] A.J. Higgins, A.P. Bruckner, in: 34th Aerospace Sciences Meeting and Exhibit, The American Institute of Aeronautics and Astronautics (AIAA), $1996 \mathrm{~Pa}$ per AIAA 1996-0342.

[15] J. Kasahara, T. Arai, S. Chiba, K. Takazawa, Y. Tanahashi, A. Matsuo, Proc. Combust. Inst. 29 (2) (2002) 2817-2824.

[16] S. Maeda, J. Kasahara, A. Matsuo, Combust. Flame 159 (2) (2012) 887-896.

[17] S. Maeda, S. Sumiya, J. Kasahara, A. Matsuo, Proc. Combust. Inst. 34 (2) (2013) 1973-1980.

[18] J.P. Sislian, R. Dudebout, J. Schumacher, M. Islam, T. Redford, J. Propul. Power 16 (2000) 41-48.

[19] K. Iwata, S. Nakaya, M. Tsue, Proc. Combust. Inst. 36 (2) (2017) 2761-2769.

[20] Y. Fang, Z. Hu, H. Teng, Z. Jiang, H.D. Ng, Aerosp. Sci. Technol. 71 (2017) 256-263.

[21] C. Viguier, A. Gourara, D. Desbordes, Symp. (Int.) Combust. 27 (2) (1998) 2207-2214.

[22] J.Y. Choi, D.W. Kim, I.S. Jeung, F. Ma, V. Yang, Proc. Combust. Inst. 31 (2) (2007) 2473-2480.

[23] J. Verreault, A.J. Higgins, R.A. Stowe, Proc. Combust. Inst. 34 (2) (2013) 1913-1920.

[24] H.H. Teng, H.D. Ng, K. Li, C.T. Luo, Z.L. Jiang, Combust. Flame 162 (2) (2015) 470-477.

[25] H.H. Teng, Z.L. Jiang, H.D. Ng, J. Fluid Mech. 744 (2) (2014) 111-128.

[26] M. Short, G.J. Sharpe, Combust. Theory Model. 7 (2) (2003) 401-416.

[27] H.D. Ng, M.I. Radulescu, A.J. Higgins, N. Nikiforakis, J.H.S. Lee, Combust. Theory Model. 9 (3) (2005) 385-401.

[28] C. Leung, M.I. Radulescu, G.J. Sharpe, Phys. Fluids 22 (12) (2010) 126101

[29] J. Tang, M.I. Radulescu, Proc. Combust. Inst. 34 (2) (2013) 2035-2041.

[30] Z.L. Jiang, Acta Mech. Sin. 20 (1) (2004) 1-15.

[31] P. Yang, H. Teng, Z.C. Jiang, H.D. Ng, Combust. Flame 193 (2018) 246-256.

[32] H.D. Ng, A.J. Higgins, C.B. Kiyanda, et al., Combust. Theory Model. 9 (2005) 159-170.

[33] J.Y. Choi, E.J. Shin, I.S. Jeung, Proc. Combust. Inst. 32 (2009) 2387-2396.

[34] H. Teng, Y. Zhang, Z. Jiang, Comput. Fluids 95 (2014) 127-131.

[35] Y. Liu, D. Wu, S.B. Yao, J.P. Wang, Combust. Sci. Technol. 187 (2015) 843-856. 